\section{COMMENT, EN PLEINE GUERRE, SE CONSTITUENT LES CHUTES D'EAU}

On parle beaucoup de la Législation de demain; on parle surtout de ce qu'elle sera par rapport à celle d'hier, et ceux qui entrevoient l'avenir s'entretiennent instinctivement de l'état actuel, comme si déjà il faisait partie du passé.

D'autres personnes plus pratiques; sentant tout le service que peut rendre encore à la Défense Nationale, à l'heure où nous écrivons, la constitution intensive des chutes d'eau (puisque personne ne peut prévoir encore la fin de la guerre), affolées par le grand nombre de décrets, de circulaires, de services compétents, nous ont demandé de préciser, plus encore en fait qu'en droit, l'état actuel de la Législation, c'est-à-dire ce que peut attendre, espérer ou... craindre des Pouvoirs publics, un industriel qui vent établir une dérivation.

Officiellement, la Législation, qui était en vigueur avant la guerre, est-elle changée?

En aucune façon : ce qu'il y a de sensiblement amélioré, c'est la routine administrative, l'état d'âme des Bureaux: pour arriver, pendant les hostilités, à la production intensive, l'Etat, en s'abstenant au moins pendant quelque temps de décourager ccux qui tendent à un résuiltat précis, doit montrer un certain désir de simplifier les rouages et ne pas commencèr par répondre négativement avant de savoir ce qui est demandé ; on semble l'avoir senti dans les sphères élevées des ministères qui détiennent une partie des richesses disponibles, et il est certain que si l'hydraulicien n'était pas arrêté par la pénurie de la main-d'œuvre, par.le prix fantastique de la moindre marchandise, il arriverait à son but plus facilement qu'autrefois, parce que les formules sont moins étroites, moins étroitement appliquées surtout; ce qui prouverait peut-être, à qui voudrait étudier la chose à fond, combien il est inutile de bouleverser une législation existante - ce qui est toujours long, grave et compliqué et comment il süffirait, dans maintes occasions, de faire aux textes de très légères modifications, si l'on pouvait rénover l'esprit qui les applique.

Aujourd'hui, les rivières publiques appartiennent toujours à l'Etat, et toutes les autres, celles qui sont régies par les articles 644 et 645 , voient toujours leurs eaux soumises au droit d'usage des riverains. Mais la nécessité vraiment nationale d'avoir de la force motrice, nous a conduits à des résultats qu'il faut analyser dans chaque catégorie.

\section{I. - RIVIËRES PUBLIQUES}

On sait que, depuis des années, traînait lamentablement dans les cartons du Luxembourg ou du Palais-Bourbon car il a fait, aller el retour, de nombreux voyages de l'un à l'autre - un projel de loi sur les usines du Domaine public dans le but officiellement proclamé de permettre anx administrations de donner des concessions de dérivation avec des cahiers des charges, de changer le vieux système des autorisations précaires et révocables sur les rivières classées, d'octrover à l'Etat des choses plus intéressantes qu'une modeste redevance, notamment de lui faire obtenir, en fin de conccssion, la prestation gratuite de ce qui aurait ćté créé dans la rivière. L'Administration supérieure, qui avait été très favorable au projet de loi, s'aperçut, peńdant les longues discussions auxquelles il avait donné lieu, que ce projet était inutile : la loi du 27 juillet $\times 87$ o lui permel de donner des concessions de canaux, avec déclaration d'utilité publique prononcée par décret sur les rivières publiques si la dérivation ne doit pas priver de l'eau une section du lit de plus de 20 kilomètres. Or, qu'est-ce qu'une chute d'cau, si cc n'est une dérivation de la rivière ayant pour but de donner non pas de l'eau qui court, mais de l'eau qui tombe, de créer non pas un chemin qui marche, mais une roue qui tourne, en produisant du courant all grand profit des régions avoisinantes?

La seule question à résoudre était de trouver le caractère d'utilité publique, afin d'obtenir un décret susceptible de faire tomber par l'expropriation les droits privés dont l'existence serait un obstacle à la réalisation de l'œuvre entreprise. On le trouva dans le fait que la distribution de l'éniergie à un tarif maximum est un bienfait national et local; la guerre survenue rendit tangible à tous cette vérité élémentaire et leva les derniers scrupules : c'est ainsi que, par décret du 25 octobrel I 9 ×4, la chute de la Basse-Isère et. par décret du Ir avril rgr6, celle du Drac inférieur ont été officiellement concédées avec déclaration d'utilité publique. Si nous prenons le texte du premier décret, tel qu'il figure au Journal Officiel du 3o octobre rgr4, nous y lisons quelques phrases à retenir, notamment les suivantes .

"Vu la demande présentée aux dates du 29 avril $1899 \ldots$ ". ce qui prouve qu'il a fallu $\mathrm{r} 5$ ans de démarche pour réussir : "le Conseil d'Etat entendu... ", ce qui indique que le haut tribunal administratif acceptait la thèse du ministère ; "sont " autorisés et déclarés d'utilité publique les travaux à entre" prendre pour une usine hydro-électrique et sa mise en vi"gueur au moyen d'un débit maximum de 270 mètres cubes "par seconde", ce qui indique le droil de faire l'application de la loi sur l'expropriation pour le canal et l'usine.

Quelque temps après, le 2 février $\mathrm{I}_{9} \mathrm{r} 7$, un décret légèrement différent était rendu, en ce qui concerne dos travaux sur la rivière de la Vienne (Papeteries de la Haye-Descartes). Ce décret ne contient pas de concession, mais une simple autorisation; seulement le concessionnaire s'engageait for mellement : $x^{\circ}$ à indemniser les tiers, riverains ou autres qui se trouveraient lésćs par l'établissemenf́ du harrage et par la surélévation des eaux ; $2^{\circ}$ à se soumettre à un cahier des charges du type adopté pour l'établissement de l'usine: hydro-électrique de la Basse-Isère (dérret du 2 octobre $\mathrm{r} 9 \mathrm{r} /$ ); $3^{\circ}$ à payer à l'Etat la redevance proportionnelle devant êtrc insérée dans le cahier des charges de la concession, comme si cette redevance était due depuis le jour de la notification du. décret d'autorisation et à payer, le cas échéant, à partir du jour de la déclaration d'utilité publique, une rederance proportionnelle au nombre de kilowatt-henres produits par l'usine (1).

(1) Celte autorisation provisotre devanl ètre changre en une concession donnée par un décret ressemblant à celui de la Basso rà̀re, est de nrafique courante aulourd'hui : l'adminstration elle-mènr l'a reconnu dans l'exposés fait par M. le ministre CLavkille, au moment nù ll a présonlés an Président de la République le décrel du 11 avril 1918, dont 11 sera parlé plus loin et qui a pour but de faciliter el d'acsonnir ioc inmalités de l'enquête próalable à la concessinn: "denmic 1914. le Ministre des "Travaux publies a décidé de ne plus laisser stahlir de grandes nsınes "sur les cours d'pau faisant partic dn Domaine public snis un régime " sutre que colui de la concession et ce, dans les comdinons anpronvics " par le Consell d'Etat pour la chute de Beaumont Vontomx (Bacse locere, "dícret du 25 octobre 1944). Pour fare cadrer co princine aver los néces" sités de la défense nationale, qu rórlame lexócution immóduate des "travanx d'amónagement des forees hydranliques, mes pridérecenrs et " moi avuns accordé ef accordons, sur la demande din Ministre de " Moi avons accordé ef accordons, sur ha demantorication provisome " l'Armement et des Fabrications de querre, lantorication provisomre "d'exécuter les travaux sans delai mais sous la reservo que le permis" sionnaire accepte explicitement de se soumeffre au riume de la "Montenx Mass solution no lifficultas, on oc " " qúcalcitrants que par la rémuisition dont leflet ne saurat depassor In a récalcitrants que par la réruisition dont dure des hostilités; il y a done intétét a lerminer an plus tôt los "formalités qui précédent la concession". 
On peul encore citer, comme décrels rendus récemment : celui du 6 août $\operatorname{Ig}_{9} 7$ concédant à la Société du Littoral Méditerranéen, avec déclaration d'utilité publiquê, l'usage de la force motrice à obtenir au moyen d'un débit maximum de 60 mètres cubes par seconde qui sera emprunté à la Durance, vers la Brillanne, par la mise en jeu de deux usines hydroélectriques, l'une déjà élablie à la Brillanne-Villeneuve, l'autre à établir au confluent du Largue dans des conditions déterminćes par une convention en date du mème jour (Journal Officiel du I2 uoût ${ }_{9}{ }^{-}$) ; celui du 6 juin $\mathrm{r} 9 \mathrm{I} 8$ (Journal Officiel du 25 juin 918 ) concédant à MM. Vuilleinoz et Tézenaz du Uontcel l'usage de la force motrice, par une dérivation de la rivière de l'Ain, obtenue sur la commune de Pont-d'Ain, pour açionner une usine hydro-électrique située sur le territoire de Priay.

L'Etat applique donc couramment aux chules d'eau la loi du 27 juillel r8 70 el a visé formellement ladite loi dans la loi de finances du 28 septembre rgr6 (arlićle r3) qui a été faite pour permeltre la participation financière de l'Etat i l'établissement d'usines hydrauliques appelées à vendre de l'énergie, el devant faire l'objel d'une concession de travaux publics. Comme l'on ne pouvait permeltre à l'Etat de faire des avances à des entreprises reposant sur des décrels dont la légalité serail discutée, on a précisé le point au-delà duquel la loi serait nécessaire, ce qui régularıse tous les décrets rendus en deçà : "les concessions sont accordées par " une loi, lorsque les travaux d'expropriation de la force " comportent le détournement des eaux de leur lit naturel " sur 'une longueur de plus de 20 kilomètres,mesurés suivanl "ce lit, ou lorsque la puissance brute dont l'usine pourra "disposer à l'étiage dépasse $\mathrm{r} 5.000$ kilowatts · lorsque les $t$ concessions seront accordées sur des cours f'eau non navi" gables ni flottables, elles ne pourraient l'être qu'après avis " conforme du Ministre de l'Agriculture.

Bien plus, comme cette loi ne permettait pas la participation financière de l'Etat à d'autres usines que celles destinées à faire la vente de l'énergic électrique, le Législateur a ćté contraint d'y revenir, pour faciliter pécuniairement les u-ines d'électro-mélallurgie ou d'électro-chimie qui sont an même degré nécessaires à la Défense Nationale, et la loi de finances du 30 juin IgI 7 a ouvert la participation de l'Etat aux usines "vendant l'énergie ou intéressant lá Défensẹ $\mathrm{Na}$ tionale et devant faire l'objet de concessions de travaux publics".

Telles sont brièvement exposées les idées que l'on peut faire valoir au point de vue de la légalité indiscutable des conceptions nouvelles.

Il nous reste à résumer ce qui a été fait au point de vue administratif, c'esl-à-dirc au point de vue : $\mathrm{r}^{0} \mathrm{de}$ la création on de la transformation de certains services ; $2^{\circ}$ des modifications à certains textes (décrets ou ,circulaires) relatifs à la constitution des chutes.

\section{a) Création ou Transformation de Services}

Avant le 14 janvier r 917 , une seule Dirẹction du Ministère des Travaux Publics était chargée des routes, de l'automobile. des usines hydrauliques et des distributions d'énergie électrique. Ledit jour, par un arrêté signé du Sous-Secrétaire d'Etat des transports, le Service central des Forces Hydranliques était créé et ne se trouvait plus annexé qu'au Scrvice des Inondations; ces deux Services sonï loin d'être incon. patibles, car le groupement est plus harmonicix que colui des automobiTes et des usines hydrauliques.

D'autre part, le 6 septembre rgr7 (Journal Officiel du ri septembre), M. Claveille, comme Sous-Secrétaire d'Etat des
Travaux Publics el des Transports, adressait à son ministre un rapport sur la nécessité de modifier, pour la durée de la guerre, le nombre et la consistance des Inspections générales des Ponts et Chaussées ; il était dit notamment : "l'im"portance, que la Houille blanche prend actuellement pour "l'avenir de la France conduit à centraliser sous l'autorité "d'un seul ingénieur, particulièrement qualifié pour diriger "ce grand service, le contròle de toules les forces hydrau" liques du territoire. C'est là une œuvre capitale ; il s'agit "de procéder à l'aménagement industriel de l'énergie con" tenue dans les fleuves el rivièr es de la France, d'organiser " et de hâter la mise en valeur. de ces forces hydrauliques, " en soumeltant loutes nos installations à un régime unifor" me et à une coordination des efforts que seul l'Etat peut "réaliser ; un service central fortement organisé doit con" trôler et activer cet aménagement et procurer ainsi dans le "plus court délai possible à l'industrie française une source " de puissance et de prospérité ".

En conséquence, par arrêté du 9 septembre 1917, M. DE LA Brosse, promü Inspecteur général des Ponts et Chaussées, était mis à la tête de ce Scrvicc.

\section{b) Modifications dé centins Textes}

Dans les anciens errements administratifs, il étail d'usage qu'un projet quelconque ne fut soumis à l'approbation supérieure que s'il était prèt, depuis le plan d'ensemble, jusques et y compris le dessin nécessaire à l'exécution du dernier détail, et si, par imprudence, le promoteur d'une entreprise déposait un projet incomplet sur certains points, le dossier faisait retour à l'envoyeur, sans que les parties étudiées puissent être utilisées, si bien qu'en pralique il n'y avait guère de différence entre un dossier ne contenant que du papicr blanc el un dossicr aúquel il n'aurait manqué qu'un dessin ou une cote. Une circulaire de M Craveille, devenu à ce moment Ministre des Tr'ıvaux publics et des Transports, a fail remarquer que l'on avait renoncé à cctte pratique, en matière de construction de roies ferrées, le projet d'exécution de tabliers métalliques ou des bâtiments n'étant présenté souvent que plusieurs années après cclui des terrassements et ouvrages d'art; pour ces motifs, le Ministre en date du r4 janvier 1918 , dans une circulaire envoyée à tous les ingénieurs, sous ce beau titre "Circulaire sur la simplification dans l'approbation des projets d'exécution ", a décidé qu'à l'avivenir les concessionnaires seront autorisés à présenter successivement les projets relatifs à l'aménagement d'unc chute, dans leur ordre naturel d'cxécution ou d'urgence, et c'est à l'ensemble de ces projels que s'appliqueront les délais prévus au cahier des charges qui devront être calculés en conséquence; de plus, les agents étaient invités à se considérer comme étant autre chose que des agents destinés à rendre compte et à faire des réserves, sur lesquelles l'Autorité ccntrale aurait à statuer ; mais à se mettre d'accord euxmêmes avec les auteurs des projets pour arrêter ceux-ci d'une façon définitive, l'intervention de l'Administration centrale n'étant plus nécessaire que si les parties en litige comportent une modification aux clanses du cahier des charges ou de l'autorisation ou si les changements proposés intéressent la sécurité publique.

Mais la modification la plus intéressante est certainement la suivante, qui nous oblige à nous arrêter un instant.

Ge qui est certain, quand on fait une chute d'eau sur le Domaine public, c'est qu'il faut travailler dans une sphère exclusivement régie jusqu'à aujourd'hui par la loi sur le régime des eaux du 8 avril 1898 . Cette loi contient un article 
ainsi conçu : "Article 43. - Toutes autres autorisations (c'est-à-dire toutes. les aulorisations pour occupations permanentes) ne peuvent être accordées que par décrets rendus après enquête, sur l'avis du Conseil d'Etat. "Mais la forme de celte enquête, la procédure à oblenir, ont élé déterminées par un décrel du $\mathrm{r}^{\mathrm{er}}$ avril $\mathrm{x}, \mathrm{0} 5$ qui oblige à certaines formalités. Or, aujourd'hui, ce n'est plus une autorisation d'occupation même permanentẹ que l'industriel sollicile: c'est une concession de travaux publics, en vertu de la loi du 27 juillet $x 870$; cettè loi aussi a ses formes obligatoires, el nolamment elle impose une enquête, plus longue, plus compliquée que celle du décret $d u r{ }^{\text {el }}$ août Igo5. A quelles formalités devra-t-on donner la préférence? A celles de cc dernier décrel ou à celles qui-sonl contenues dans l'ordonnance royale du $\mathbf{8}$ févricr 1834 à laquelle, implicilement, se réfère la loi du 27 juillel 1870 . Soyez assurés que si l'on n'avait pas solutionné par une réponse définitive la question posée, l'esprit admimstralif auraul imposé les deux fornualıtés successivement, ce qui n'est pas une simplification... Aussi l'()fficiel du 17 avril r9I8 (page 3297) publie-l-il un décrel jort intéressant en date du i a avril. Ce décret a-t-il ćté pris dans la limıle de la compélence du Président de la République 'Cela n'est pas douteux, car une ordonnance royale, comme celle de r834, el un décret, comme celui du $x^{\text {er }}$ août sgo5, sont des actes du Pouvoir exécutif; et, d'après un principe incontestable, ce Pouvoir peut toujours légalement refaire le texte qu'il a déjà composé, alors même qu'il l'aurait signé en verlu d'une délégation de la loi, parce quo rette délégation est permanente.

Pour bien comprendre la fusion des deux formalités en une seule, il faudrait analyser l'une et l'aułre, tout d'abord, e! la troisième ensuite, ce qui serait un peu long: qu'importe d'ailleurs le passé si aujourd'hui nous saisissons bien ce qu'il faut faire? Ia lecture du décret suffirail à elle scule pour nous renseigner. Examinons cependant les diftérentes phases qui se développent devant nous.

a) En premier lieu une demande doit être faite, énonçant les noms du cours d'eau, des départements et communes intéressés, et - on reconnaîtra ici la préocoupation constante de ne pas troubler les installations antéricures - la nomenclature des établissements placés immédiatement en amont et en aval ; à cette demande, qui doil être rédigée sur papier timbré, est annexé un dossier sur papier libre, donl les différentes pièces sont énumérées à l'arlicle 4.

Dans les huil jours au plus tard, le Ministre en accuse réception (ce qui est un progrès, le demandeur sait au moins si le dossier ne s'est pas égaré) el désigne l'Ingénieur en chef qui doit ctre saisi de l'affaire ; il est probable qu'en cas de plusicurs départements intéressés, celui du département dans lequel les ouvrages doivent être plus particulièrement importants sera désigné.

Co fonctionnaire a un délai d'un mois pour donner son avis au Ministra qui décide si l'affaire doit être suivic ou classée; cst-il besoin de dire que l'avis du Ministre, neuf fois sur dix. ne sera que la reproduction de celui de l'Ingénieur? Le Ministre informe le demandeur qui doit, dans le délai de deux mois, fournir à l'Ingénieur désigné, tous renseignements techniques, avec calcul à l'appui, et un projet de cahier des charges. Le dossier est étudié complètement par l'Ingénieur qui rédige ses propositions tendant à la mise à l'enquête, et, en cas do demandes concurrentes, indique colle qui paraît susceptible d'etre relenue avec les raisons qui militent en faveur de cette décision; le dossier repart alors pour Paris, est soumis par le Ministre au Conseil supé- rieur des Travaux publics et c'est là que sa demande recoit sa consécration définitive; elle est acceptée - et, dans ce cas, la mise à l'instruction proprement dite va immédiatement commencer - ou est rejetée; Je demandeur el l'Ingénieur sont avisés de la décision (1).

b) La mise à l'instruction comprend quatró formalités distincles: l'enquête qui a pour but de provoquer les observations du... public; on sait ce que l'on entend par ce terme vague; il comprend surtout, ainsi que le connaissent bicn tous ceux qui ont assisté à des événemenls de cet ordre, le défilé des ennemis systímatiques du projet el de son auteur ; théoriquement il devrail comprendre tous ceux qui, au point de' vue général de la région intéressée, salubrité, hygiène, protection contre les accidents, ont des remarques à faire; il faut que le dossier resle déposé dans toutes les communes riveraines du cours d'eau, depnis la limite amont du remous jusqu'à l'extrémité du canal de fuile.

La visite des lieux par l'Ingénieur . formalilé qui a pour but de recevoir les observalions plutòt lechniques de ceux dont les intérêts pourraient être compromis directement par l'exécution du projet : relèvemenl du plan d'eau qu gènerait une industrie d'amont, débordement du cours d'eau sur des propriétés privées, etc.

Les conférences administratives dont liutılité esl de provoquer les observalions des grands services: Navigation, Hydraulique agricole, Posles et Télégraphes; ces services doivent recevoir communication du dossier et donner leurs observations dans le délai d'un mois, passé lequel, s'ils n'ont formulé aucune objection, i]s sont considérés comme adhérents.

La consultation des corps spéciaux, parmi lesquels il faut citer : le Conseil général (en application de la loi du 26 décembre rgo8) ou de la Commission départementale à qui délégation aura été donnée de ce chef, les Conseils municipa'ux des communes intéressées, les Chambres de commerce.

La clôture de l'instruction osl faite par l'íngénieur en chef qui rédige un rapport, donne son avis el deux mois après la clôture de l'enquête envoie le tout a'u Ministre des Travaux publics qui consulte le Conseil supérieur des Travaux publics et prend l'avis du Ministre des Finances.

c) La déclaration d'utilité publique esl prononcée el, s'il y a lieu, la concession accordéc, soil par décrel rendu en la forme d'un règlement d'administration publique, soit par une loi, en application des dispositions de la loi du 27 juillet $\mathrm{x} 870$.

Nous croyons avoir suffisamment expliqué Je régime actuel de l'obtention du décret, quand nous aurons appelé l'altention du lecteur, sur ce fail que tout ce qui n'est qu'autorisation même permanente, ef non pas demande de concession administrative en la forme de concession de travaux publics, doit rester soumis an seul décret du ${ }^{\text {er }}$ ant 1905 .

(1) On ne sera pas étonn' de voir dans le décret que chaque formalité loit être faite dans un déla donné, c'est une inlication excellente, qui permet all. Ministre et même aux Ingénieurs de donner des rappels, basée sur la loi, et non pas sur leur propre impalience, s'lls ne reçolvent pas les réponses qu'ils ont" sollicitées, mais il ne faut, pas c rolro qu'une nullité quelconque pourrait servir de sanction à l'inobservation les délais. Quel serait d'ailleurs le Tribunal qu appliquerait cette sanclion; ce n'est pas le Tribunal judiciare, car bien quil lu appartienne de rrohercher si la déclaration d'utilité devait ètre faite par une loi ou un décret, il ne peut vérifier la régularité intrinsèque de cel acte, il doit s'assurer que la déclaralıon d'utilite publique s'applique bien aux travaux pour le-quels l'expropriation est requise ; mais il ne peut contester le caractère d'utilite publique quilu est attribué (Cas. 28 jum 1897 D.98 23) n'examiner dans quellos mesures l'expronriation ost nécessaire à leur exécution, ni apprécier leur opportunité (22 janvier 1901, Cas I) 1892183. If ne peut refuser le prononeer l'exprofiriation par le molif que les ronditions de déla imposées pour colle-ci n'auraient pas été observées (mp̂me arrâ) Voir Dir Dallo\% No Expropriation jo 34 . Quant au Conseil d'Etat, 11 n'annule le décret que si des formules nécessaires à la rósularlté de l'observation ont été omises. C. E. lor juin $1849 \mathrm{et} 11$ juin 1880 


\section{II. - GOURS D'EAU NON NAVIGABLES NI FLOTTABLES}

Observations sur l'Etat actuel. Facilités nouvelles

Nous n’avons pas besoin de répéter qu'à l'époque actuelle l'Administration a sur ces rivières un simple pouvoir de surveillance, au point de viue des entreprises qui pourraient compromettre la sécurité, la salubrité et les intérêts génćraux d'une région; elle l'exerce au moment où elle donne "lautorisation de barrage", qu'il s'agisse soit d'une chute en dérivation, soit d'une chute en refoulement; l'Etat n'est point sur son domaine et la voie normale de son intervention ne peut donc pas être une concession, comme pour la catégorie précédente, car on ne concède que ce-qui vous appartient. "nemo dat quod non habet". Sans doute, si l'Etat justifie d'une utilité publique, il peut favoriser une entreprise par la déclaration de cette utilité, au moyen d'un décret (qui permet d'obtenir, gràce ì l'expropriation, le droil de passage dans le sol ou le sous-sol des propriélés privées, l'indemnité résultant de la suppression des droits d'eau étant réglée, conmie un dommage provenant d'un travail public, par une décision du Tribunal administratif. Mais, en dehors de cette déclaration, qui ne peut s'attacher qu'au caractère de l'œuvre entreprise, l'industriel doit faire à l'amiable l'acquisition des droils d'aqueduc en sous sol ou en surface, ainsi que celle des droits à l'usage de l'eau. Sans doule, pendant la période des hostilités, il a été pratiqué, à cause de l'utilité nationale qui s'altache à l'exécution rapide des Iravaux, certaines réquisitions de terrain, le propriétaire se trouvant dépossédé manu militari ; mais comment l'industriel propriétaire se tirera-t-'il de cette situation délicate, quant, à la cessation des hostilités, la cause initiale de la réquisition c'est-à-dire l'utılité nationale, s'évanouira dans les douceurs de la paix?

C'est pourquoi on ne saurait trop regretter que la modification si modérée et si juste, appelée par les vœux les plus ardents des industriels et réclamée par eux, n'ait jamais été admise par le Parlement. Il aurait suffi, au lieu de repnendre depuis les fondations jusqu'au faîte, une législation qui a fait ses preuves, de la compléter par une simple assimilation de l'Industrie à l'Agriculture en ce qui concerne les servitudes d'aqueduc et d'appui, prononcées avec attribution d'une indemnité par le Tribunal civil. Il y aurait, sans injustice, ni chantage, ni trouble pour l'avenir une prise de possession, justifiée par l'intérêt général qui commande, surtout par les temps que nous traversons, de considérer la production industrielle comme au moins aussi utile que la production agricole.

Quant aux facilités données aux entreprises intéressant la Défense nationale, l'Administration y a pourvu, soit par les mesures provisoires de la réquisition, soit par des subventions accordées à certaines usines (avec un remboursement obligatoire dans un délai de deux ans). On comprend 'qu'il nous soit impossible de donner unc énumération quelconque des cnireprises ayant bénéficié d'une pareille favour

(1) 11 est évident que l'on ne doit pas confondre.ces subventions octroyées à des entreprises déterminées arcc les subventions générales budgétares contenues dans la loi des Finances du 29 min 1918, fournal O/ficiel du 30 jum) Nous y lisons: titre premier, Ftat A. Tableau par ministère ef par chapitre des dépenses lu hudpel orlmare des services civils de loxorcice 1918: Ministère les Travaux Publics, dépenses extraordinares Travaux (no 96 et suivants). Service des forces hydrauligues trals gínéraux de liureanx $30000 \mathrm{fr}$, subventions et publications diverses $30000 \mathrm{fr}$. Etudes et recherches scientifiques de laboratoire, Subvention aux établıssements scientıfiques : $440000 \mathrm{fr}$. Travaux préparatoires 1000.000 Ftablissements d'usines par l'Etat: subientions ou avances pour constructions d'usines : 1.000 .000 L'article 4 de la lol des finances du 29 jum 1917 contenait déjà un crédit de 4.500000 francs qui n'a pas été utilisé en entier, sauf erreur de notre part.
Indépendamment de ces faveurs particulières, il faut reconnaître que, d'une manière générale, l'Administration pendant la guerre a essayé de simplifier ses services, et de diminuer les délais qui étaient jusqu'alors imposés aux demandes d'autorisation de barrage.

Comme pour la catégorie précédente, nous allons examiner les modifications dans les services et les simplifications dans les formalités.

\section{a) Modifications dans les Services}

Un Décret du 22 décembre $\mathrm{r} 9 \mathrm{r} 6$, rendu sur la demande de deux Ministres (celui de l'Agriçulture, M. Cuémentel, celui de l'Armement et des Fabrications de guerre, M. Albert Tnomas) et paru dans le Journal Officiel du 23 décembre I916, a transféré pour la durée des hostilités au Ministène de l'Armement lés attributions confiées au Mınistère de l'Agriculture en ce qui concerne l'aménagement et l'utilisation des forces hydrauliques sur les cours d'eau non navigables et flottablles. Le Ministère de l'Armement prenail le 3o octobre $\mathrm{r}^{1} \mathrm{r} 7$, un arrêté pour raltacher à son Service dit des "Forces motrices" tout ce qui concerne les cours d'eau non navigables pour décharger un autre Service dit des Fabrications de guerre qui se trouvait surchargé outre rnesure.

Mais le décret du 22 décembre $\mathbf{9} 96$, pour des raisons qu’il est inutile de faire connaître fut trouvé par les deux Ministères intéressés, celui de l'Agriculture et de l'Armement, comme trop général, et une scission, d'un commun accord, fut opérée à la date du $x^{\text {er }}$ février $x_{91} 8$.

Le Ministère de l'Agriculture garde dans son domaine les chutes inférieures en puissance à $500 \mathrm{kws}$ ét celles qui regardent la traction électrique sur voie ferrée ; le Ministère de l'Armement conserve toutes les autres chutes, c'est-à-dire célliles qqui, supérieures à $500 \mathrm{kw}$, n'ont aucun rapport avec la traction. Nous pouvons voir, en effet, une conséquence de cette attribution. Dans un décret signé le $\mathrm{I}^{\text {er }}$ juin $\mathrm{Ig}$ I8, (Journal Officiel du 4 juin igr8) par M. PoIncaré, relativiement à l'utilisation des eaux du lac d'Orédon, pour augmenter dans les Hautes-Pyrénées la puissance de l'usine productive d'Eget - ce qui touche seulement l'électrification des chemins de fer de la Compagnie du Midi - nous ne trouvons pas la signature du Ministre de l'Armement, mais seulement celle du Ministre de l'Agriculture.

\section{b) Simplification des Formalités}

Par une circulaire du 27 février Ig $^{18}$, de M. Je Ministre de l'Armement aux préfets, les instructions sủivantes ont été données : il est surtout rappelé que par une circulaire du 2 juillet 1908 , les Préfets doivent, toutes les fois qu'ils sont saisis d'une demande de dérivation susceptible de produire plus de cent poncelets, la communiquer au Ministre (l'intervention du Ministre, en juillet r 908 dans une ailiaire purement locale, avait été considérée comme nécessaire pour qu'il puisse, paraît-il, surveiller de Paris, les intérêts généraux de la région considérée). Le Ministre d'aujourd'hui ordonne que cette communication lui soit faite d'urgence, mais iI explique le motif de cette urgence : " il ne s'agit pas de " subordónner à ces renseignements, dit-il, l'ouverture de "l'instruction ni l'autorisation administrative, mais ils me " permettront d'apprécier l'intérêt de la Défense nationale " et les mesures dans lesquelles je devrais, en raison de cet" intérêt, intervenir pour aplanir les difficultés qui entra"veraient une solution rapide". 
Plus loin dans la même circulaire, le Ministre indique que : "l'instruction continuera à être faite dans les termes " du titre $X I$ du décrel du $\mathrm{I}^{\text {or }}$ août $\mathrm{Ig}$ g5. Mais la durée ac" tuelle de l'instruction, qui alteint parfois plusieurs mois, " et même plus d'une année, devra être considérablement ré" duite. J'estime qu'entre le dépôt d'une demande en régle" mentation de barrage accompagnée de toutes les justifica" tion:s nécesisaires, el le moment où vous m'adresserez le " dossier de l'affiaine par application du paragraphe 2 de " l'article 14 du décret de igo5, il ne devra pas s'écouler un " laps de lemps supérieur à quatre mois. En cas d'impossi" bilité d'arriver à ce résultat, vous aurcz à m'adresser un " rapport sommaire justifiant cette dérogation à mes pres"criptions ct indiquant le délai nécessaire dans l'espèce.

"Pour arriver à ce résultat, je vous signale notamment " qu'il y a lieu „d'accélérer tout ce qui concerne la consul" tation des diffénents Services publics intéresséz. Dans ce " but on demandera aux pétitionnaures les documents néces" saires pour qque ces Services soient saisis parallèlement dès " l'origine de l'affaire. Les Ingénieurs ne devront pas perdre " de vue que ces Services, chargés d'autres tâches parfois " aussi urgentes, ne sont pas toujours aussi bien placés " qu'eux pour apprécier l'intérêt du développement rapide " de l'énergie hydraulique, et que, souvent, il suffira d'une "certaine insistance et de rappels verbaux pour aplanir " leurs objections et hâter leur avis. En cas d'insuccès, ils " vous en rendront compte aussitôt et vous devrez faire " cesser le retard ou me demander d'intervenir auprès de "l'Administration compétente ".

Il nous a semblé que, par le temps qui court, les indications qui précèdent, méritaient d'être données.

Paul Bougault, Avocat à la Cour d'appel de Lyon.

\section{LE PREMIER OFFICE RÉGIONAL D'ÉNERGIE ÉLECTRIQUE (CRÉÉ A MARSEILLE)}

Le 7 mai igi8, le Comiré d'Action Economique de la $X V^{\ominus}$ RÉGron ( $\left.{ }^{1}\right)$ a tenu une séance plénière du Procès-Verba] de laquelle nous extrayons ce qui suit touchant la création d'un Office régional d'Energie dont notre éminent collaborateur M. René Tavernier a montré le rôle nécessaire dans son article sur "Le Projet de loi relatif à l'utilisation de l'Energie électrique et les Services "publics". La Houille Blanche, $\mathrm{n}^{\circ}$ I 48 (Septembre-Octobre $19 \mathrm{q} 7$ ).

M. Gavoty, Président de 1"Union des Syndicats agricoles des Alpes et de Provence, expose les motifs qui l'ont engagé à demander l'inscription à l'ordré du jour de la séance plénière du Comité, de la question de l'institution d'Offices régionaux d'Encrgie électrique. L'emploi de cette énergie par

(1) Ces "Comités d'Actıon économique de Régions " sont la réalisation d'un Vou émis par " l'Association française pour le développement des Travaux publics " au Congrès de 1900. - Ce sont des Commissions lacales qui dans toute la France sont réparties survant les Régions milllarres. Y sout représentés l'Elat, les Services des Ponts et Chaussées, des Amóliorations agricoles, des Eaux et Forêts - le Service de l'Inspection des Forges, pendant la guerre - les Consells généraux, les Chambres da Commerce, les Syndicats industriels, les Sociétés d'Agriculture Ces "Comités consultatifs d'Action économique " crés par le Décret du 20 octobre 1915 , ont pour mission de donner leur avis sur loutes los initiatives propres à dévolopper la richesse publique et à ćtudier les projets répondant à ce but. Ils ont par leur organisation et leur indépendance, un rôle d'une très grande efticacité dans la solution des conflits d'intéréts si nuisibles à la prospérité natıonale. l'agriculture a préoccupé de tous temps les associations agricoles. Plusieurs mois avant la guerre, I Union des Alpes et de Provence avait mis la question à l'élude el ses délégués dquaient assister au Congrès de la houille blanche fixé au mois de septembre rgr4, à Lyon. La guerre a empêché le Congrès, mais non la continuation des études. Elle a même donné une importance nouvelle à la question de mise en valeur des forces hydro-électriques, car, si toules les installations avaient été effectúés avant le conllil actuel, elles auraient été d'un appoint considérable. Mais il n'est pas trop tard pour bien faire el il sagit maimtenanl, pour chaque branche de l'activité nationale, d'étudıer ses propres besoins en énergie électrique et de chercher, par une entente entre intéressés, notamnıent entre Industrıels el Agriculteurs, à réaliser cette utilisation avec méthode et dans los meilleures condilions pratiques.

L'Agriculture a toujours demandé qu'il soit tenu complix de ses besoins lors de l'aménagement des cliutes d'eau et de la création des usines hydro-électrıques et que des tarifs peu élevés lui soient consentis. Ces principes sont reconnus ct réalisés par le projet de loi déposé le 27 juillel rg×7. Ce projet admet que, toutes les fois qu'il sera question d'aménager une chute d'eau sur un cours d'eau du domaine public, il y aura à faire intervenir les besoins de l'Agriculture en eau et en énergie.

Pour profiter de ces avantages, il faut que les agriculteurs soient représentés par un organe autorisé : Syndicat ou Groupement de syndicats. D'autre part, pour justifier les tarifs de faveur demandés par les agriculteurs, il faul que, par une entente avec l'Industrie, ces derniers arrivent à utiliser l'énergie électrique dans certaines conditions de saisons et d'heures qui permettent l'emploi le plus rationnel de cette force et en abaissent le prix de revient. C'est pour préparer ces accords qu'une étude en commun s impose dans une région comme la Provence qui est plus apte que d'autres à cmployer l'énergie électrique, non seulement pour les Irrigations, mais pour les usages de la ferme et de l'Industrie. M. Gavoty ajoute que, dans une réunion présidée par lui à Lyon, le is mars igr8, plusieurs représentants de syndicats agricoles de la région intéressée par les irrigations se sont rencontrés avec des industriels et des ingénieurs et ont discuté ces questions; mais ils ont estimé qu'aucune assemblée n'était plus qualifiée que le "Comité d'Action Economique de la $X V^{\ominus}$ Région " pour jeter les bases d'une entente entre l'Industrie et l'Agriculture, entente indispensable si l'on veut arriver à un résultat pratique. Cela explique la mise à l'ordre du joûr de la séance plénière dúu Comité, de la question d'utilisation des forces hydro-électriques el de création d'offices d'énergie. M. Gavory estime que, de l'exposé que va faire de celte question M. René TAvernier, Inspecteur général dez Ponts cl Chaussées en retraite, qui connail admirablement la matière, il résultera, ainsi que des échanges de vue motivés par cet exposé, des résolutions qui permettront, en aidant à l'utilisation pratique de l'énergie électrique, d'aider à la reprise et au développement du travail national après la guerre.

\section{RAPPORT DE M. RENÉ TAVERNIER}

Inspecteur général des Ponts et Chaussées en retraile

Dans l'exposé qui m'est demandé, je laisserai complètement de côté les considérations de doctrine, les aperçus a priori. Je ne m'appuierai que sur des faits dûment constatés. Parmi ces faits, il y en a deux essentiels, qui caractérisent l'évolution des entrepries: de troneport ol te distri- 